\title{
SEARCH SPACE EXPLORATION USING LEVY FLIGHT WITH TURNING ANGLE CONSTRAINT AND BOUNDARY REFLECTION
}

\author{
M. H. A. MAJID and M. R. ARSHAD \\ School of Electrical \& Electronic Engineering, Universiti Sains Malaysia (Engineering Campus), \\ 14300 Nibong Tebal, Penang, Malaysia \\ E-mail: helmi_mjd@yahoo.com,eerizal@usm.my \\ www.usm.my
}

\begin{abstract}
Source searching is an important task in many real world applications and it has been widely studied using various types of robotic systems. In this task, the robot has to first detect the presence of the source in the search space before it can continue to locate the source to its approximate location. In order to efficiently detect the source, the robot must optimize search space exploration. Lévy flight is one of the optimal algorithms for search space exploration. However, for a destructive type of source searching, Lévy flight is susceptible to two problems. Firstly, the possibility of immediate returns to the previously visited site is high. Secondly, robot tends to temporarily stuck at the boundary of the search space until a new angle that bring robot away from the boundary is generated. In this paper, the solutions to the two problems are proposed by imposing angle constraints to Lévy flight and by using boundary reflection, reflectively. The results show that the exploration capability of the Lévy flight with the proposed strategy is improved compared to the conventional Lévy flight model.
\end{abstract}

\section{Introduction}

Robotics has enormous potential for real world applications and tasks, especially the one that is not possible or inefficient to be performed by humans. In general, different types of task that suit robotics systems (e.g. single or multiple autonomous robots) can be classified as tasks that are dangerous to human, task that require area coverage, tasks that demand simultaneous deployment and task that require redundancy [1]. Source searching or source seeking is one of the tasks that falls within the classification. In real world scenarios, source searching and localization is an important task in many applications such as in a search and rescue operation, environmental monitoring, detection of chemical leakage, mine countermeasures, searching for a flight recorder (black box) and scientific studies.

In a real source searching problem, there are three possible scenarios can occur due to robot limited sensing range and intensity decaying property of the source. Firstly, there is a possibility that the source is not detected as soon as the robot is deployed into the search space. In this scenario, the robot must rely on an algorithm which is independent of source intensity to optimize search space exploration in order to optimize detection time. Secondly, there is possibility that the intensity of the source is directly detected once robots are deployed into the search space. In this case, an algorithm which exploits source intensity measurement must be used to optimize convergence speed and accuracy of convergence. In the third scenario, robots do not detect the source once they are deployed, but the source may become detected after robots explore the search space and vice versa. In this case, independent algorithms for both detectable and undetectable source signals must be considered. As a result, a complete and optimal source searching algorithm must be able to work in both situations where source signal is detected and source signal is not detected. Currently, this issue has not 
been thoroughly studied where in most current studies it assumed that source signal is always detected or a nonoptimal random movement is considered when source is not detected [2-4]. As a result, the overall efficiency source searching is not optimal because the time taken to locate the source not only influenced by the time taken to locate the source once detected but it is also influenced by the time taken to detect the source. In this study, the issue of source detection for the individual robot is considered which can be considered as a complimentary algorithm for the existing source searching algorithm such as algorithm based on Particle Swarm Optimization (PSO) [5], Glowworm Swarm Optimization [6], Ant Colony Optimization [7], etc.

Animals including insects and microorganisms employ different types of random movement as searching and hunting strategies. This type of movement is employed when preys or food sources are not located within their sensing range [8]. Random movement is also employed when available information about the source location is not sufficient to assist searching [9]. In addition, microorganisms have no adequate sensing capability to allow them to directly detect and sense food sources from a far distance. This limitation makes them rely heavily on random walk (RW) to detect the source intensity before they can locate the source site by following the gradient [10]. Thus, in order to maximize chances of detection to avoid starvation and to optimize energy consumption, an optimal random search strategy is adopted by many types of animals [11].

In biological studies, several types of RW have been used to model animal random searching and foraging strategies. However, Lévy flight (LF) is the most efficient random searching strategy for many animal species [12]. For examples, LF has been used to model pelagic birds foraging pattern, animals including human hunting strategy and organisms food searching pattern [13]. In general, LF is formed by the combination of short (i.e. local exploration) and long steps length (i.e. global exploration during the transition between two local sites). As a result, the area explored by LF is larger compared to other types of RW for an equal number of steps. Thus, LF permits the individual searcher to visit a new search site which have not been previously visited and minimize frequency of revisiting a similar search site [14]. The global and local exploration characteristics of the LF increase the chances of a searcher to locate the source by balancing the two exploration strategies.

However, for a destructive type of source searching (i.e. a source only need to be located only once), Lévy flight is susceptible to two problems from the perspective of the individual robot. Firstly, the possibility of immediate returns to the previously visited site is high during local exploration due to randomly generated turning angle. Secondly, robot tends to temporarily stuck at the boundary of the search space until a new angle that bring robot away from the boundary is generated. As a result, the exploration capability of the robot is not optimal. In this study, a reliable strategy to eliminate the two problems is proposed to improve exploration capabilities of the robot.

\section{Lévy Flight Model}

In search space exploration, LF is used to generate waypoints need to be followed by a robot in order to explore the search space. The FL consists of two parameters which characterized its exploration characteristics, namely flight length and turning angle. Based on Figure 1, the next waypoint, $\boldsymbol{p}_{\boldsymbol{k}}=\left(x_{k}, y_{k}\right)$ generate by LF at the $k^{\text {th }}$ update step can be calculated by

$$
\begin{aligned}
& x_{k}=x_{k-1}+l_{k} \cos \left(\phi_{k}\right) \\
& y_{k}=y_{k-1}+l_{k} \sin \left(\phi_{k}\right)
\end{aligned}
$$


in which $x_{\min } \leq x_{k} \leq x_{\max }$ and $y_{\min } \leq y_{k} \leq y_{\max }$ where $\left[x_{\min }, y_{\min }\right]$ and $\left[x_{\max }, y_{\max }\right]$ are the search space boundaries, $l$ is the step length and $\phi$ is the absolute turning angle of LF. The turning angle can also be expressed as a relative turning angle, $\varphi$ given by

$$
\varphi_{k}=\phi_{k}-\phi_{k-1}
$$

The turning angles are sampled from a continuous uniform distribution, $\phi \in P(\phi) \longleftarrow$ $U\left[\phi_{\min }, \phi_{\max }\right]$ defined by the following probability density function (PDF)

$$
P(\phi)=\left\{\begin{array}{lll}
\frac{1}{\phi_{\max }-\phi_{\min }} & \text { if } & \phi \in\left[\phi_{\min }, \phi_{\max }\right] \\
0 & \text { if otherwise }
\end{array}\right.
$$

The range of the angles can be either be $\phi \in[-\pi, \pi]$ or $[0,2 \pi]$. The distribution of the LF flight length can be approximated as a power-law distribution representing a truncated Lévy-stable distribution is given by $[15,16]$ :

$$
P(l) \approx l^{-\mu} \text { for } 1<\mu \leq 3
$$

where $\mu$ is the scaling factor. Notice that for $\mu \leq 1$ and $\mu>3$ motion is no longer Lévy instead it becomes ballistic motion and Brownian motion, respectively.

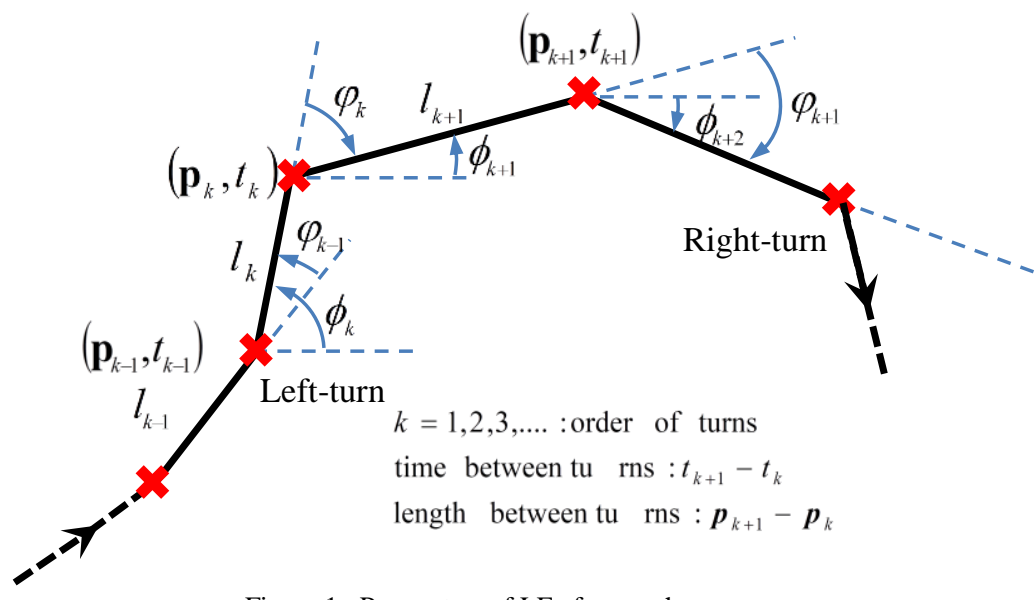

Figure 1. Parameters of LF of a searcher

\subsection{Angle Constraint}

To improve exploration efficiency of the previous LF model, turning angle can be constrained by implementing a lower limit as shown in Figure 2. As shown in the Figure 2, an LF with angle constraint has better exploration as shown by the shaded cells illustrated in Figure 2(b) compared to LF without angle constraint as indicated by the shaded cells shown in Figure 2(a) for a two successive steps. Thus, the turning angles of the LF can be constrained within a range of $\theta_{\min } \leq \theta \leq \pi$ to optimize exploration and at the same time maintain local exploration capability of the LF. The angle $\theta$ in term of absolute turning angle of two successive steps is 


$$
\theta=|\pi-| \phi_{k+1}-\phi_{k}||
$$

A relationship between absolute turning angles between two successive LF paths is shown Figure 3. A new constrained waypoint, $\boldsymbol{p}_{\boldsymbol{k}+1}$ due to $\theta$ limit can be expressed as

$$
\mathbf{p}_{\mathbf{k}+1}=\mathbf{p}_{\mathbf{k}}+l_{k}\left[\begin{array}{l}
\cos \left(\phi_{k, \text { new }}\right) \\
\sin \left(\phi_{k, \text { new }}\right)
\end{array}\right]
$$

where the new turning angle expressed as turning limit are given by

$$
\phi_{k, \text { new }}=\left\{\begin{array}{lll}
\phi_{k}-\operatorname{sign}\left(\pi-\left|\phi_{k}-\phi_{k-1}\right|\right) \Delta \theta_{k, k-1} & \text { if } & \phi_{k-1}<\phi_{k} \\
\phi_{k}+\operatorname{sign}\left(\pi-\left|\phi_{k}-\phi_{k-1}\right|\right) \Delta \theta_{k, k-1} & \text { if } & \phi_{k-1} \geq \phi_{k}
\end{array}\right.
$$

where $\Delta \theta_{k, k-1}=\theta_{\min }-\theta_{k}$ and $0 \leq \theta_{\min } \leq \pi / 2$. Thus, by considering this angle, the possibility of immediately returning to the previously searched site is minimized.

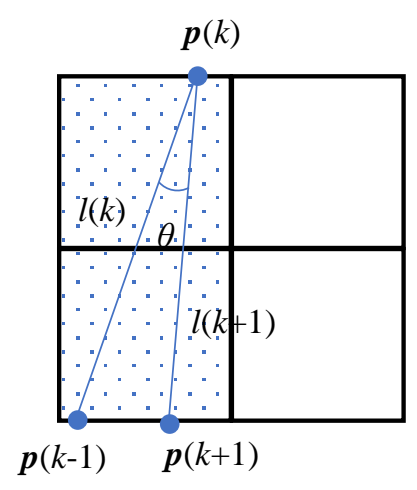

(a)

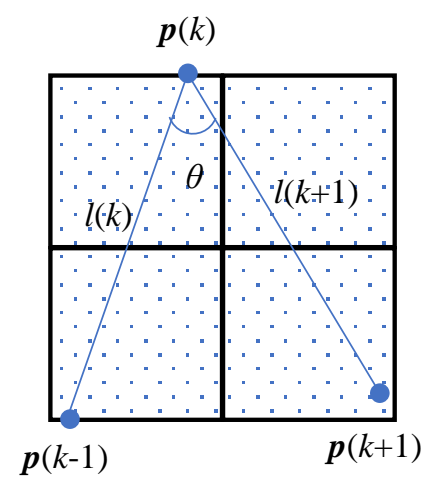

(b)

Figure 2. Impact of angle constraint (a) LF without angle constraint, $\theta<\theta_{\min }$ (b) LF with angle constraint, $\theta \geq \theta_{\min }$

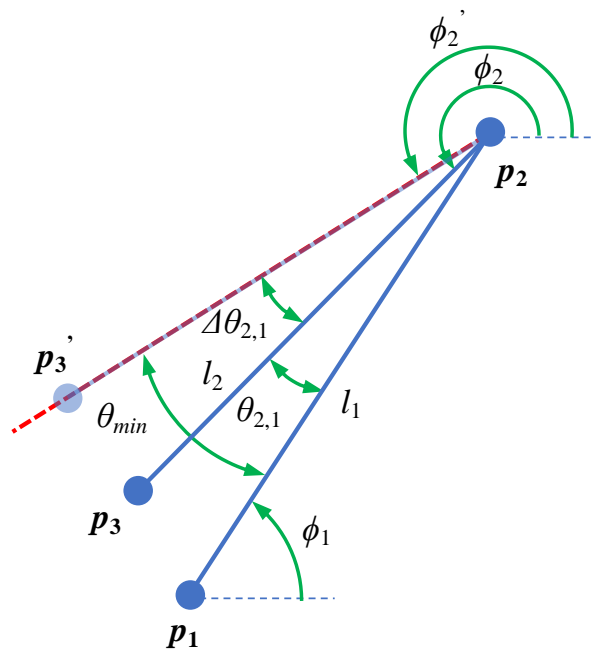

Figure 3. Turning angle constrained to minimize immediate return to the currently visited sites 


\subsection{Boundary Reflection}

Another issue is the problem at the boundary of search space where robots may have to wait at the boundary for several iterations before the uniformly randomly generated angle bring the robot away from the boundary (i.e. due to invisible path) as illustrated in Figure 4. To solve this issue, robot path can be reflected as shown in Figure 4. From the figure and based on the vector summation approach,

$$
\begin{aligned}
& \hat{\mathbf{d}}_{k, k+1}=\mathbf{N}\left(\hat{\mathbf{d}}_{k-1, k} \cdot \mathbf{N}\right)+\mathbf{a} \\
& \hat{\mathbf{d}}_{k-1, k}+\mathbf{a}=\mathbf{N}\left(\hat{\mathbf{d}}_{k-1, k} \cdot \mathbf{N}\right)
\end{aligned}
$$

where $\hat{\mathbf{d}}$ is the unit vector and $\boldsymbol{N}$ is the unit normal vector to the search space boundary. Solving (9) for a and substitute into (8) gives

$$
\hat{\mathbf{d}}_{k, k+1}=2 \mathbf{N}\left(\hat{\mathbf{d}}_{k-1, k} \cdot \mathbf{N}\right)-\hat{\mathbf{d}}_{k-1, k}
$$

Thus, from Figure 3, the reflected path can be expressed as

$$
\mathbf{p}_{k+1}=\mathbf{p}_{k}+l_{k+1} \hat{\mathbf{d}}_{k, k+1}
$$

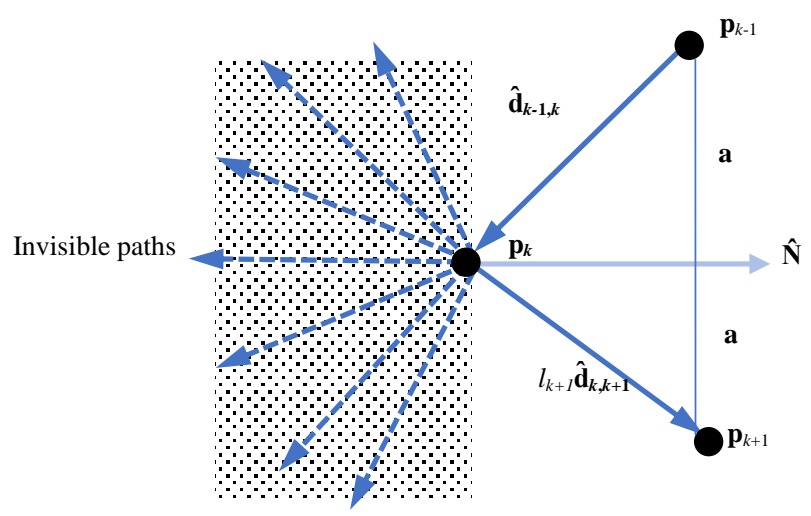

Figure 4: Invisible LF path and the corresponding path reflection at the search space boundary

\section{Result and Discussion}

In this study, the LF parameters are set to be $\mu=1.1$ and $l_{\min }=1.5 \mathrm{~m}$ for a $25 \times 50 \mathrm{~m}^{2}$ search space with detection radius, $r_{d}=6 \mathrm{~m}$. Based on these values, the performance of the proposed LF modification can be evaluated. Since the source searching process considered in this study is a destructive search, it is necessary to minimize the possibility of revisiting a similar site in a search space which has been recently visited to improve exploration of the individual robot. Figure 5 illustrates Percent Area Coverage (PAC) (see Appendix) difference between LF with and without angle constraint (i.e. $\mathrm{PAC}_{\text {angle_constraint }}-\mathrm{PAC}_{\text {angle_nonconstraint }}$ ). The PAC of LF with angle constraint is improved compared to LF without angle constraint. The larger the value of $\theta_{\min }$ the greater the improvement is observed. This is because a large value of $\theta_{\min }$ reduces the possibility of the robot to immediately revisit the previously searched area (i.e. the grid cell used for PAC computation) and thus, exploration of a new search site is improved. However, the improvement of the PAC is relatively small (i.e. up to $3.8 \%$ when $\theta_{\min }=90^{\circ}$ ) because the robot is only restricted not to return to the previously searched area in a two successive steps 
but it may possibly return to the same site after several iterations. In general, this trend can be observed for different sizes of search space, but with decreasing PAC for a similar number of maximum iteration used for simulation.

In terms of Frequency of Visiting (FoV) (see Appendix), larger $\theta_{\text {min }}$ means smaller FoV because the robot is capable of exploring a new search space which has not been recently explored. The impact of $\theta_{\min }$ on the FoV improvement is demonstrated through difference of FoV between LF with and without angle constraint (i.e. FoV $\mathrm{V}_{\text {angle_constraint }}-F_{\text {o }} V_{\text {angle_nonconstraint }}$ ) as depicted in Figure 6. The negative value means FoV of the LF without angle constraint is larger compared to FoV of the LF with angle constraint. From the figure it can be observed that the larger the value of $\theta_{\text {min }}$, the better the improvement of the LF. However, since according to definition the FoV is independent of search space size, the corresponding value of FoV does not significantly change as the search space size increases. The improvement of LF through the combination of angle constraint and boundary reflection can clearly be observed in Figure 7. The PAC of LF agent with angle constraint (AC) and boundary reflection (BR) is better than a pure $\mathrm{LF}$ where $\mathrm{LF}+\mathrm{AC}+\mathrm{BR}$ reaches certain PAC level faster (i.e. less iterations) compared to $\mathrm{LF}$ as can be observed in the figure. For example, LF achieves $98 \%$ PAC at $k=2000$ while LF with AC and BR achieved 90\% PAC for the same number of steps. The shaded region represents the range of PAC values for the entire number of runs. Thus, it is clearly proven that the proposed LF with angle constraint and boundary reflection successfully improved exploration of the individual robot. Figure 8 shows an example of the implementation of the proposed LF modification for 5 different robots deployed from the same initial position, but different initial orientation where the source detection radius is assumed to be $r_{d}=6 \mathrm{~m}$ and located at $(0,20)$.

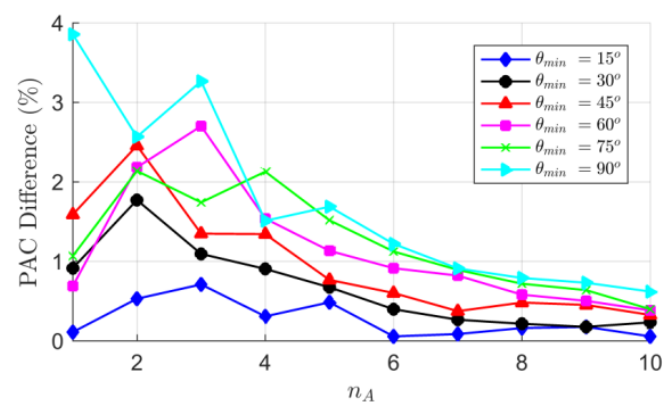

Figure 5: PAC for different values of $\theta_{\min }$ in different sizes of search space using optimal LF parameters for $25 \mathrm{~m}$ by $50 \mathrm{~m}$ search space

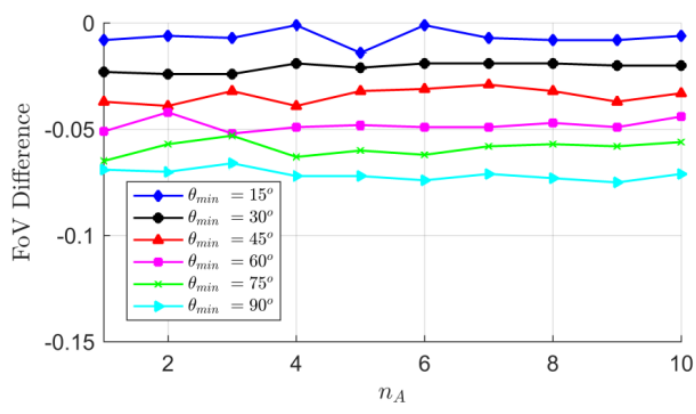

Figure 6: The average FoV for different values of $\theta_{\min }$ in different sizes of search space using optimal LF parameters 


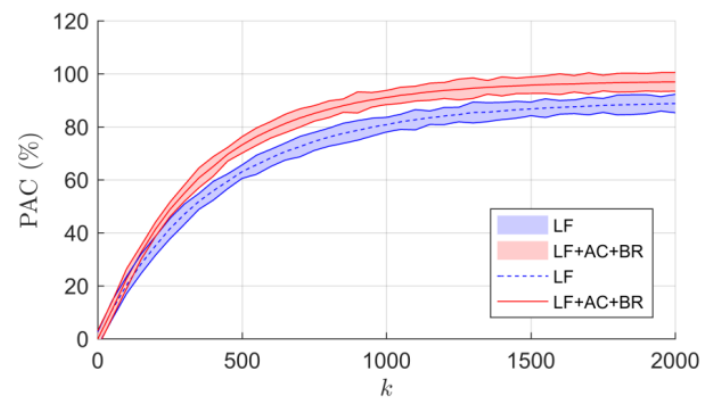

Figure 7: The average of 1000 runs number of steps, $k$ taken to reach certain value of PAC for pure LF and LF with angle constraint (AC) and boundary reflection (BR)

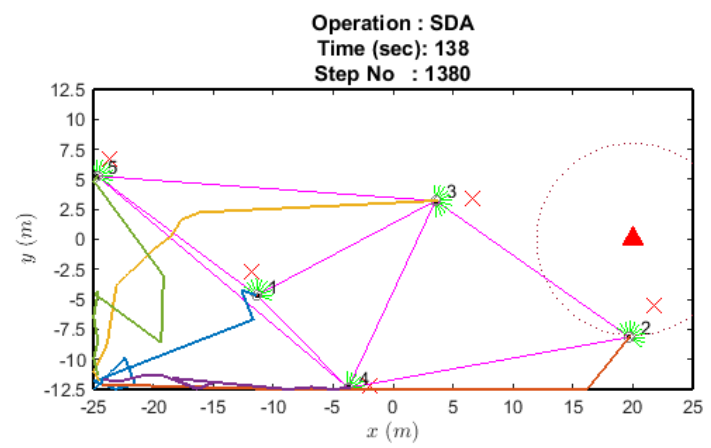

Figure 8: Robot trace during source detection with $\mu=1.1, l_{\min }=1.5 \mathrm{~m}, \theta_{\min }=15^{\circ}$ and $r_{d}=6 \mathrm{~m}$. Trace indicator: blue $\left(\mathrm{R}_{1}\right)$, red $\left(\mathrm{R}_{2}\right)$, yellow $\left(\mathrm{R}_{3}\right)$, purple $\left(\mathrm{R}_{4}\right)$, green $\left(\mathrm{R}_{5}\right)$, pink (inter-robot communication link)

\section{Conclusion and Future Work}

This study proposed turning angle limit and boundary reflection for LF in order to optimize the search space exploration capability of the robot. The results show that the proposed strategy successfully improved exploration capability of the robot with better PAC and FoV. In the future, the improvement of LF for better exploration capability considering multiple robots will be studied.

\section{Acknowledgments}

This research is funded by the Fundamental Research Grant Scheme (FRGS). Account No.: 1001/PELECT/6071346.

\section{Appendix}

The PAC and FoV are defined as follow:

$$
P A C=\frac{N G_{v i s}}{N G_{t o t}} \times 100 \%
$$

where $N G_{v i s}$ is the number of visited and $N G_{t o t}$ is the total number of grids divisions of search space.

$$
F o V_{a v g}=\frac{1}{N G_{v i s}} \sum_{i=1}^{N G_{v i s}} F o V_{i}
$$

where $F_{o} V_{i}$ is the frequency of visiting of grid $i$. 


\section{References}

1. Tan, Y., and Zheng, Z.-y.: 'Research Advance in Swarm Robotics', Defence Technology, 2013, 9, (1), pp. 18-39.

2 Melo, R., Junior, C.J.S., Albuquerque, C.V.E., and Oliveira, S.C.: 'An Performance Analysis of a PSO-Based Algorithm for Swarm Robotics', in Editor (Ed.)(Eds.): 'Book An Performance Analysis of a PSO-Based Algorithm for Swarm Robotics’ (2018, edn.), pp. 66-71.

3 Kumar, A.S., Manikutty, G., Bhavani, R.R., and Couceiro, M.S.: 'Search and rescue operations using robotic darwinian particle swarm optimization', in Editor (Ed.)(Eds.): 'Book Search and rescue operations using robotic darwinian particle swarm optimization' (2017, edn.), pp. 1839-1843.

4 Husni, N.L., Handayani, A.S., Nurmaini, S., and Yani, I.: 'Cooperative searching strategy for swarm robot', in Editor $(\mathrm{Ed} .)^{\wedge}(\mathrm{Eds}$.$) : 'Book Cooperative searching strategy for swarm$ robot' (2017, edn.), pp. 92-97.

5 Greenhagen, C., Krentz, T., Wigal, J., and Khorbotly, S.: 'A real-life robotic application of the particle swarm optimization algorithm', in Editor (Ed.): 'Book A real-life robotic application of the particle swarm optimization algorithm' (2016, edn.), pp. 1-5.

6 Senanayake, M., Senthooran, I., Barca, J.C., Chung, H., Kamruzzaman, J., and Murshed, M.: 'Search and tracking algorithms for swarms of robots: A survey', Robotics and Autonomous Systems, 2016, 75, Part B, pp. 422-434.

7 Ferreira, M.V.M., Fonseca, J.P.S., and Tavares, J.J.P.Z.S.: 'Attabot: Open Platform Inspired on Brazilian Ants for Swarm Robots', in Editor (Ed.): 'Book Attabot: Open Platform Inspired on Brazilian Ants for Swarm Robots' (2018, edn.), pp. 225-229.

8 Humphries, N.E., and Sims, D.W.: 'Optimal foraging strategies: Lévy walks balance searching and patch exploitation under a very broad range of conditions', Journal of Theoretical Biology, 2014, 358, pp. 179-193.

9 Bartumeus, F., and Catalan, J.: 'Optimal search behavior and classic foraging theory', Journal of Physics A: Mathematical and Theoretical, 2009, 42, (43), pp. 434002.

10 Li, L., Nørrelykke, S.F., and Cox, E.C.: 'Persistent Cell Motion in the Absence of External Signals: A Search Strategy for Eukaryotic Cells', PLOS ONE, 2008, 3, (5), pp. e2093.

11 Viswanathan, G.M., Buldyrev, S.V., Havlin, S., da Luz, M.G.E., Raposo, E.P., and Stanley, H.E.: 'Optimizing the success of random searches', Nature, 1999, 401, (6756), pp. 911-914.

12 Bartumeus, F., da Luz, M.G.E., Viswanathan, G.M., and Catalan, J.: 'Animal Search Strategies: A Quantitative Random-walk Analysis', Ecology, 2005, 86, (11), pp. 30783087.

13 Raichlen, D.A., Wood, B.M., Gordon, A.D., Mabulla, A.Z.P., Marlowe, F.W., and Pontzer, H.: 'Evidence of Lévy walk foraging patterns in human hunter-gatherers', Proceedings of the National Academy of Sciences, 2014, 111, (2), pp. 728-733.

14 Chang-Yong, L., and Xin, Y.: 'Evolutionary programming using mutations based on the Levy probability distribution', IEEE Transactions on Evolutionary Computation, 2004, 8, (1), pp. 1-13.

15 Viswanathan, G.M., Afanasyev, V., Buldyrev, S.V., Havlin, S., da Luz, M.G.E., Raposo, E.P., and Stanley, H.E.: 'Lévy flights in random searches', Physica A: Statistical Mechanics and its Applications, 2000, 282, (1-2), pp. 1-12.

16 Sutantyo, D.K., Kernbach, S., Levi, P., and Nepomnyashchikh, V.A.: 'Multi-robot searching algorithm using L'evy flight and artificial potential field', in Editor (Ed.): 'Book Multi-robot searching algorithm using L'evy flight and artificial potential field' (2010, edn.), pp. 1-6. 